Please do not remove this page

RMIT

UNIVERSITY

\title{
Observation of nonlinear surface waves in modulated waveguide arrays
}

Xinyuan, Qi; Garanovich, Ivan; Zhang, Zhiyong; Sukhorukov, Andrey; Krolikowski, Wieslaw; Mitchell, Arnan; Zhang, Guoquan

https://researchrepository.rmit.edu.au/esploro/outputs/9921864189601341/filesAndLinks?institution=61RMIT_INST\&index=null

Xinyuan, Q., Garanovich, I., Zhang, Z., Sukhorukov, A., Krolikowski, W., Mitchell, A., Zhang, G., Nashev, D., \& Kivshar, Y. (2009). Observation of nonlinear surface waves in modulated waveguide arrays. Optics Letters, 34(18), 2751-2753. https://doi.org/10.1364/OL.34.002751

Document Version: Published Version

Published Version: https://doi.org/10.1364/OL.34.002751

Repository homepage: https://researchrepository.rmit.edu.au

(C) 2009 Optical Society of America

Downloaded On 2023/04/27 00:06:46 +1000

Please do not remove this page 


\title{
Observation of nonlinear surface waves in modulated waveguide arrays
}

\author{
Xinyuan Qi, ${ }^{1,2, *}$ Ivan L. Garanovich, ${ }^{1}$ Zhiyong Xu, ${ }^{1}$ Andrey A. Sukhorukov, ${ }^{1}$ Wieslaw Krolikowski, ${ }^{1}$ \\ Arnan Mitchell, ${ }^{3}$ Guoquan Zhang, ${ }^{2}$ Dragomir N. Neshev, ${ }^{1}$ and Yuri S. Kivshar ${ }^{1}$ \\ ${ }^{1}$ Nonlinear Physics Centre and Laser Physics Centre, Research School of Physics and Engineering, \\ Australian National University, Canberra, ACT 0200, Australia \\ ${ }^{2}$ The Key Laboratory of Weak Light Nonlinear Photonics, Ministry of Education, Nankai University, \\ Tianjin 300457, China \\ ${ }^{3}$ School of Electrical and Computer Engineering and Centre for Ultra-high Bandwidth Devices for Optical Systems \\ (CUDOS), Royal Melbourne Institute of Technology University, Melbourne, VIC 3001, Australia \\ *Corresponding author: qixycn@gmail.com
}

Received June 9, 2009; accepted July 17, 2009; posted August 18, 2009 (Doc. ID 112547); published September 9, 2009

We describe theoretically and study experimentally nonlinear surface waves at the edge of a modulated waveguide array with a surface defect and a self-defocusing nonlinearity. We fabricate such structures in a $\mathrm{LiNbO}_{3}$ crystal and demonstrate the beam switching to different output waveguides with a change of the light intensity due to nonlinear coupling between the linear surface modes supported by the array.

(C) 2009 Optical Society of America

OCIS codes: $190.0190,240.6690,190.6135$.

The study of surface waves in periodic photonic structures such as photonic crystals or optical lattices is attracting increasing attention. Their basic properties have many similarities to the physics of electrons localized at crystalline surfaces, which were first discussed by Tamm [1] and Shockley [2]. The direct observations of such states have been performed only in photonic systems $[3,4]$, such as periodic waveguide arrays. It was found that strong surface localization is possible when the edge waveguide is modified and the defect strength exceeds a certain threshold, in agreement with original predictions by Tamm [1]. Such a surface defect can also be induced through optical nonlinearity, resulting in the formation of surface lattice solitons [5-11]. On the other hand, it was recently demonstrated that arrays of periodically curved waveguides can support a novel type of linear surface modes without any surface defects $[12,13]$.

In this Letter, we describe theoretically and observe experimentally nonlinear surface waves at the edge of modulated waveguide arrays. We demonstrate nontrivial beam dynamics due to an interplay between three mechanisms of surface localization: (i) waveguide bending, (ii) fabricated surface defect, and (iii) nonlinear beam self-action. First, we present the theoretical classification of linear surface modes supported by the curved arrays with a surface defect. Next, we demonstrate experimentally that nonlinear beam self-action can provide effective control of the output beam profile, including switching between different waveguides near the surface.

We fabricate arrays of modulated waveguides by titanium indiffusion in a 50 -mm-long $X$-cut $\mathrm{LiNbO}_{3}$ crystal with defocusing photorefractive nonlinearity [14], featuring a transverse refractive index profile containing a negative surface defect (lower refractive index value at the first waveguide) [see Fig. 1(a)]. We choose the waveguide bending profile composed of sinusoidal sections, $x_{0}(z)=A[\cos (4 \pi z / L)-1]$ for $0<z$
$<L / 2, x_{0}(z)=-x_{0}(z-L / 2)$ for $L / 2<z<L$, and $x_{0}(z)$ $=x_{0}(z-L)$ for $z>L$ [see sketch in Fig. 1(a)]. Here $x_{0}$ is the transverse waveguide shift, $z$ is the longitudinal coordinate, and $A$ and $L$ are the waveguide axes bending amplitude and period, respectively. Two curved arrays with different bending amplitudes $(A$ $=21.5 \mu \mathrm{m}$ and $A=24.5 \mu \mathrm{m})$ are fabricated. Both samples contain one full bending period of $L$ $=50 \mathrm{~mm}$.

It was shown that, away from the boundaries, the linear beam diffraction in a curved array appears the same after each bending period as in a straight array with the effective coupling coefficient $C_{e}$ [15-18]. For our bending profile, $C_{e}=C J_{0}\left[\xi A / A_{0}\right]$, where $C$ is the

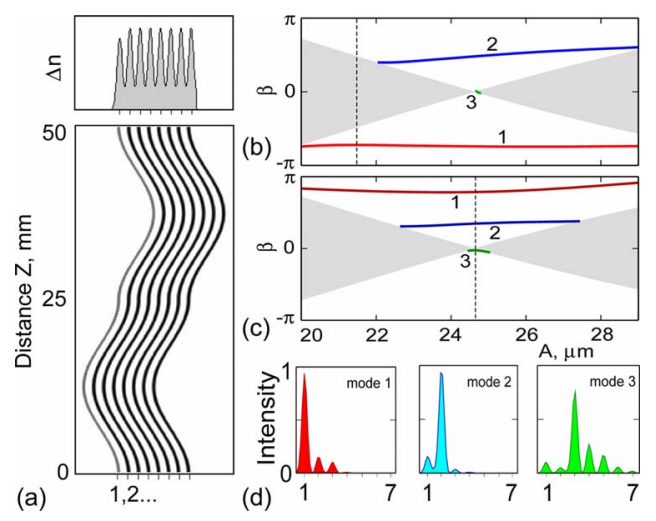

Fig. 1. (Color online) Linear surface modes in a curved waveguide array with a surface defect. (a) Schematic of the array (bottom) and index change (top). (b), (c) Propagation constants of linear surface modes versus waveguide bending amplitude $A$ for surface defects (b) $\rho_{1}=-0.52$ and (c) $\rho_{1}=-0.56$. Gray shading marks the array transmission band, and the vertical dashed line corresponds to (b) $A$ $=21.5 \mu \mathrm{m}$ and (c) $A=24.5 \mu \mathrm{m}$. (d) Intensity profiles at $z$ $=0, L$ of three different surface modes for $A=24.5 \mu \mathrm{m}$, corresponding to mode dispersion lines marked 1-3 in (c). 
coupling coefficient in the absence of bending [12], $J_{0}$ is the Bessel function of first kind of order $0, \xi$ $\simeq 2.40$ is its first root, $A_{0}=\xi \lambda L / 8 \pi^{2} n_{0} d=24.6 \mu \mathrm{m}, \lambda$ $=532 \mathrm{~nm}$ is the laser wavelength used in the experiments, $n_{0}=2.35$ is the refractive index of $\mathrm{LiNbO}_{3}$ substrate, and $d=14 \mu \mathrm{m}$ is the spacing between the centers of the adjacent waveguides. Note that the average beam diffraction is completely suppressed $\left(C_{e}=0\right)$ for a set of bending amplitudes including $A$ $=A_{0}$, analogous to the effect of dynamic localization for electrons in crystals with an applied ac bias field [16].

The curved waveguide arrays can support linear surface modes even in the absence of surface defects [12]. We consider here a more general case when the edge waveguide is modified [Fig. 1(a)]. We use coupled-mode equations to model the beam dynamics $\quad[12,16], \quad i \mathrm{~d} a_{n} / \mathrm{d} z+C \exp \left[-i \dot{x}_{0}(z)\right] a_{n+1}$ $+C \exp \left[i \dot{x}_{0}(z)\right] a_{n-1}+\rho_{n} a_{n}+\gamma\left|a_{n}\right|^{2} a_{n}=0$, where $a_{n}(z)$ are the normalized mode amplitudes, $n=1,2 \ldots$ is the waveguide number, and $a_{n \leqslant 0} \equiv 0$ is due to the structure termination. The value of $\rho_{1}$ defines the detuning of the surface waveguide, while $\rho_{n>1} \equiv 0$. We take the value of the normalized nonlinear coefficient $\gamma=$ -1 to account for the defocusing photorefractive nonlinearity of $\mathrm{LiNbO}_{3}$. By matching the experimentally measured discrete diffraction pattern in a straight waveguide array, we determine the value of the coupling coefficient $C \simeq 0.09 \mathrm{~mm}^{-1}$. By measuring the beam propagation close to the boundaries of the curved arrays we estimate the defect strengths as $\rho_{1}=-0.52$ in the fabricated sample with $A=21.5 \mu \mathrm{m}$ and $\rho_{1}=-0.56$ for the sample with $A=24.5 \mu \mathrm{m}$.

First, we perform theoretical analysis of the linear surface modes, extending the methods described in [12]. We characterize the mode dispersion with the phase $\beta$ accumulated over one modulation period and present the mode tuning on the modulation amplitude in Figs. 1(b) and 1(c) for both parameter values of $\rho_{1}$. We find that the profiles of the three fundamental modes (marked 1-3) are similar for $\rho_{1}=-0.56$ [shown in Fig. 1(d)] and $\rho_{1}=-0.52$ (not shown) owing to their common physical origin. Mode 1 is supported by the surface defect, similar to Tamm states [5]; it exists for a wide range of bending amplitudes and has a profile with intensity maximum at the first waveguide [Fig. 1(d), left]. Modes 2 and 3 exist owing to waveguide bending, similar to the defect-free surface states [12]. The input and output mode profiles (at $z=0, L$ ) have intensity maxima at the second and third waveguides, respectively [Fig. 1(d), middle and right].

Next, we simulate the beam dynamics under the effect of nonlinear self-action, when light is coupled to a single waveguide number $n$ at the input with the normalized intensity $I=\left|a_{n}(0)\right|^{2}$. We show the simulated beam evolution in an array with $A=24.5 \mu \mathrm{m}$ in Fig. 2. In Figs. 2(a)-2(d) we excite the first waveguide $(n=1)$. At low powers, in the essentially linear propagation regime, the first (defect) surface mode [Fig. $1(d)$, left] is excited. At higher input powers, the defocusing nonlinearity increases the strength of the

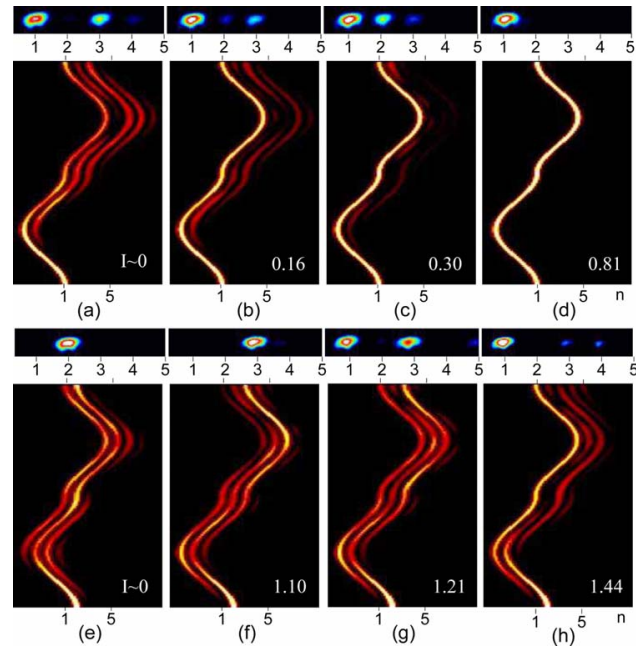

Fig. 2. (Color online) Surface waves in a modulated waveguide array $(A=24.5 \mu \mathrm{m})$. In each block: top images, experimental results for different illumination times; bottom images, numerical simulations. In (a)-(d) the beam is launched into the first waveguide; in (e)-(h) the second waveguide is excited. (a), (e) Linear propagation: input power $1 \mu \mathrm{W}$; illumination time $5 \mathrm{~s}$. In (b)-(d) the illumination times are 0.50, 1.25, and $4.25 \mathrm{~min}$; in (f)-(h) the illumination times are 10.92, 17.67, and $18.42 \mathrm{~min}$, respectively. Input power in (b)-(d) and (f)-(h) is $4 \mathrm{~mW}$. In the numerical calculations the normalized input intensity $I$ is marked in the corners.

negative defect in the first waveguide, and eventually the entire beam becomes trapped in the first waveguide [see Figs. 2(c) and 2(d)].

In Figs. 2(e)-2(h) we excite the second waveguide $(n=2)$. At low light intensity, the second surface mode [Fig. 1(d), middle] is excited. However, at higher intensity, nonlinear coupling and interaction between different linear modes is present. We note that even away from the surface, the nonlinear beam dynamics is highly nontrivial at these power levels [19-22]. We observe switching of the output beam position between the second, third, and first waveguides as we increase the intensity [Figs. 2(f) and 2(g)]. As the input intensity grows further, the beam becomes localized at the edge waveguide [Fig. 2(h)], indicating the formation of a self-trapped nonlinear surface wave. At even higher intensities (not shown) the nonlinearity completely detunes the input channel, and the light becomes trapped back to the second waveguide.

In our experiments, we use the beam from a cw la$\operatorname{ser}(\lambda=532 \mathrm{~nm})$. The beam is ordinary polarized, perpendicularly to the plane of the array, to minimize bending losses and radiation. The nonlinear refractive index change in the photorefractive $\mathrm{LiNbO}_{3}$ depends on the input power and slowly increases with illumination time. We therefore monitor the output intensity distribution onto a CCD camera with increasing illumination until a steady state is reached. We focus the beam to the first and second waveguides and for each case show the output intensity profiles at four different illumination times in Figs. 2(a)-2(h), top images. We observe that as the nonlinear response increases, the output beam switches between the different waveguides. There is good agreement 


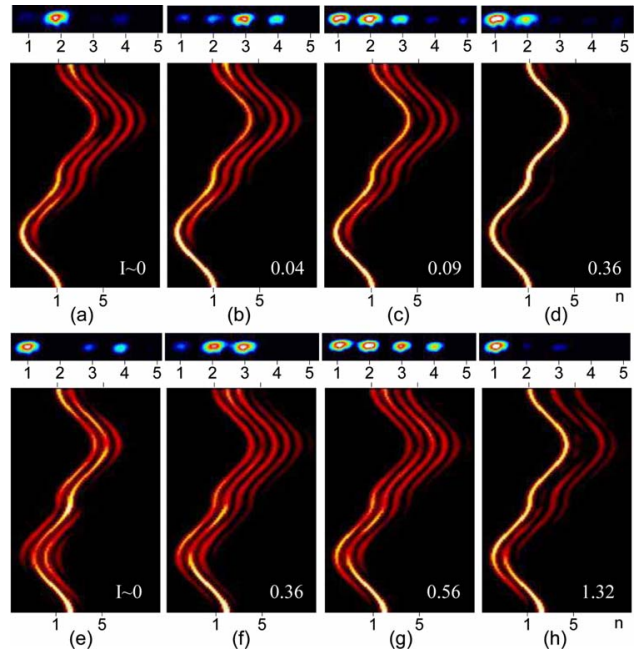

Fig. 3. (Color online) The same as in Fig. 2 but for $A$ $=21.5 \mu \mathrm{m}$. (a), (e) Linear propagation: power $1 \mu \mathrm{W}$; illumination time $5 \mathrm{~s}$. In (b)-(d) and (f)-(h) input power is $4 \mathrm{~mW}$. In (b)-(d) illumination time is $0.33,4.50$, and $7.33 \mathrm{~min}$; in (f)-(h) illumination time is $1.00,3.08$, and $13.33 \mathrm{~min}$, respectively.

between the experiments and numerical simulations, except at very high input powers, when we register a slow oscillatory beam motion between the first two waveguides owing to the charge dynamics in photorefractive crystal, which is not described by the coupled-mode equations.

Figure 3 shows numerical and experimental results for the second sample with bending amplitude $A=21.5 \mu \mathrm{m}$. In this case a band of extended linear states coexist together with a single linear surface mode [see dashed line in Fig. 1(b)]. Therefore we also identify the presence of leaky surface modes, which remain quasi localized for our sample length. Beating between all these modes determines beam propagation in the linear regime, as shown in Figs. 3(a) and $3(\mathrm{e})$. As we increase the input powers/illumination times, we observe complex beam reshaping and switching, which depends on the nonlinearity strength; see Figs. 3(b)-3(d) and Figs. 3(f)-3(h). We note that in Fig. 3(b) the experimental intensity distribution is affected by the transient beam dynamics owing to the short illumination time.

In conclusion, we have studied surface modes in modulated waveguide arrays, which demonstrate the features of both optical Tamm states and discrete surface solitons. We have shown that the interplay of different surface modes types enables novel means of light shaping and switching between different output waveguides.
This work was supported by the Australian Research Council.

\section{References}

1. I. E. Tamm, Z. Phys. 76, 849 (1932).

2. W. Shockley, Phys. Rev. 56, 317 (1939).

3. P. Yeh, A. Yariv, and A. Y. Cho, Appl. Phys. Lett. 32, 104 (1978).

4. N. Malkova, I. Hromada, X. S. Wang, G. Bryant, and Z. G. Chen, Opt. Lett. 34, 1633 (2009).

5. K. G. Makris, S. Suntsov, D. N. Christodoulides, G. I. Stegeman, and A. Hache, Opt. Lett. 30, 2466 (2005).

6. S. Suntsov, K. G. Makris, D. N. Christodoulides, G. I. Stegeman, A. Hache, R. Morandotti, H. Yang, G. Salamo, and M. Sorel, Phys. Rev. Lett. 96, 063901 (2006).

7. Y. V. Kartashov, V. A. Vysloukh, and L. Torner, Phys. Rev. Lett. 96, 073901 (2006).

8. G. A. Siviloglou, K. G. Makris, R. Iwanow, R. Schiek, D. N. Christodoulides, G. I. Stegeman, Y. Min, and W. Sohler, Opt. Express 14, 5508 (2006).

9. E. Smirnov, M. Stepic, C. E. Ruter, D. Kip, and V. Shandarov, Opt. Lett. 31, 2338 (2006).

10. C. R. Rosberg, D. N. Neshev, W. Krolikowski, A. Mitchell, R. A. Vicencio, M. I. Molina, and Yu. S. Kivshar, Phys. Rev. Lett. 97, 083901 (2006).

11. W. H. Chen, Y. J. He, and H. Z. Wang, Opt. Express 14, 11271 (2006).

12. I. L. Garanovich, A. A. Sukhorukov, and Yu. S. Kivshar, Phys. Rev. Lett. 100, 203904 (2008).

13. A. Szameit, I. L. Garanovich, M. Heinrich, A. A. Sukhorukov, F. Dreisow, T. Pertsch, S. Nolte, A. Tunnermann, and Yu. S. Kivshar, Phys. Rev. Lett. 101, 203902 (2008).

14. M. Matuszewski, C. R. Rosberg, D. N. Neshev, A. A. Sukhorukov, A. Mitchell, M. Trippenbach, M. W. Austin, W. Krolikowski, and Yu. S. Kivshar, Opt. Express 14, 254 (2006).

15. H. S. Eisenberg, Y. Silberberg, R. Morandotti, and J. S. Aitchison, Phys. Rev. Lett. 85, 1863 (2000).

16. S. Longhi, M. Marangoni, M. Lobino, R. Ramponi, P. Laporta, E. Cianci, and V. Foglietti, Phys. Rev. Lett. 96, 243901 (2006).

17. I. L. Garanovich, A. A. Sukhorukov, and Yu. S. Kivshar, Phys. Rev. E 74, 066609 (2006).

18. R. Iyer, J. S. Aitchison, J. Wan, M. M. Dignam, and C. M. de Sterke, Opt. Express 15, 3212 (2007).

19. V. V. Konotrup, O. A. Chubykalo, and L. Vazquezet, Phys. Rev. E 48, 563 (1993).

20. D. Cai, A. R. Bishop, N. Gronbech-Jensen, and M. Salerno, Phys. Rev. Lett. 74, 1186 (1995).

21. I. L. Garanovich, A. A. Sukhorukov, and Yu. S. Kivshar, Opt. Express 15, 9547 (2007).

22. A. Szameit, I. L. Garanovich, M. Heinrich, A. Minovich, F. Dreisow, A. A. Sukhorukov, T. Pertsch, D. N. Neshev, S. Nolte, W. Krolikowski, A. Tunnermann, A. Mitchell, and Yu. S. Kivshar, Phys. Rev. A 78, 031801 (2008). 